\title{
Characterization of non-small cell lung cancer transforming to small cell lung cancer and its response to EGFR-TKI: a case report
}

\author{
Guotao Fang ${ }^{1 \#}$, Weiwei Liu ${ }^{1 \#}$, Yanhong Shang ${ }^{1,2}$, Ran Huo ${ }^{1}$, Xiaoliang Shi ${ }^{3}$, Yanan Wang ${ }^{1}$, Jinghua $\mathrm{Li}^{4}$ \\ ${ }^{1}$ Department of Medical Oncology, Affiliated Hospital of Hebei University, Baoding, China; ${ }^{2}$ Hebei Key Laboratory of Cancer Radiotherapy and \\ Chemotherapy, Baoding, China; ${ }^{3}$ OrigiMed Co. Ltd, Shanghai, China; ${ }^{4}$ Medical Office, Affiliated Hospital of Hebei University, Baoding, China \\ "These authors contributed equally to this work. \\ Correspondence to: Jinghua Li. Medical Office, Affiliated Hospital of Hebei University, 212 East Yuhua Road, Baoding 071000, China. \\ Email: lijinghua56789@163.com.
}

\begin{abstract}
Epidermal growth factor receptor-tyrosine kinase inhibitors (EGFR-TKIs) have demonstrated significant survival benefits for advanced non-small cell lung cancer (NSCLC) patients with sensitive EGFR mutations. However, patients with EGFR-TKI treatment often develop acquired resistance subsequently. Transformation from NSCLC to small cell lung cancer (SCLC) is a rare EGFR-TKI resistance mechanism for patients with sensitive EGFR mutations. Herein, we report a NSCLC patient with EGFR exon 19 deletion treated with EGFR-TKI. During treatment, the pathological type of tumor showed transformation from NSCLC to combined SCLC and then to pure SCLC after acquiring EGFR-TKI resistance. Genomic analysis revealed that the EGFR exon 19 deletion, TP53 Y220H mutation, and retinoblastomal transcriptional corepressor 1 (RB1) F755V mutation existed persistently. Immunohistochemical results showed the loss of EGFR and RB1 expression in SCLC. The patient received multi-line chemotherapy with platinum agents and experienced a briefly effective window, but died of aggressive tumor progression. We profiled the transformation from NSCLC to SCLC of this case and pointed out the importance of repeat biopsy in response to EGFR-TKI resistance. Our results showed a novel RB1 F755V mutation which may be associated with RB1 loss. This report summarized the clinical characteristics, mechanisms, and predictors of SCLC transformation, and discussed the treatment after transformation.
\end{abstract}

Keywords: Adenocarcinoma; small cell lung cancer (SCLC); epidermal growth factor receptor (EGFR); acquired resistance; case report

Submitted Nov 05, 2021. Accepted for publication Jan 14, 2022.

doi: 10.21037/atm-21-6991

View this article at: https://dx.doi.org/10.21037/atm-21-6991

\section{Introduction}

Epidermal growth factor receptor $(E G F R)$ is one of the most important oncogenes in non-small cell lung cancer (NSCLC) (1). Epidermal growth factor receptor-tyrosine kinase inhibitors (EGFR-TKIs) have been shown to prolong the survival of patients with EGFR mutations, but acquired resistance often develops within 10 14 months (2). Exchange of tumor subtypes may be a possible mechanism acquired resistance, such as previously reported transformation from adenocarcinoma to squamous cell carcinoma and from NSCLC to small cell lung cancer
(SCLC) is a possible mechanism of acquired resistance $(3,4)$. Therefore, it is necessary to re-biopsy the growing tumor or recurrent site to understand the acquired resistance to EGFR-TKI treatment. Studies have showed that TP53 inactivation and RB1 loss might be potential mechanisms of SCLC phenotype conversion after TKI resistance $(5,6)$. However, the mechanism of acquired drug resistance in patients treated with EGFR-TKI is still not clear. Herein, we report a complex case of transformation from adenocarcinoma to SCLC after EGFR-TKI treatment; highlight the profile of transformation from NSCLC to SCLC; summarize the clinical characteristics, 
Table 1 The profiling of gene mutation in four pathological biopsies

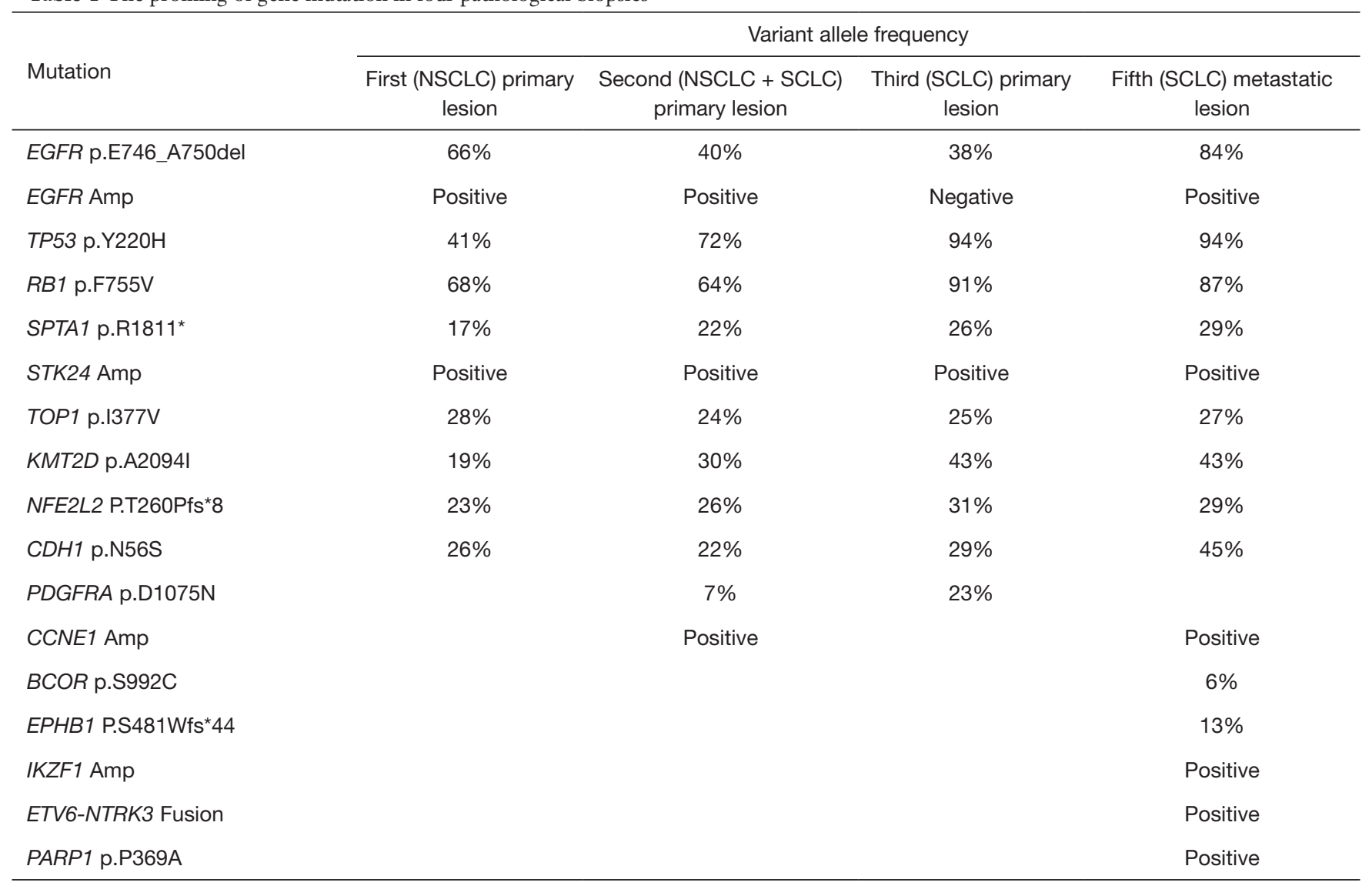

NSCLC, non-small cell lung cancer; SCLC, small cell lung cancer.

mechanisms, and predictors of SCLC transformation; and discuss the treatment after transformation. We present the following article in accordance with the CARE reporting checklist (available at https://atm.amegroups.com/article/ view/10.21037/atm-21-6991/rc).

\section{Case presentation}

In June 2015, a 44-year-old man was diagnosed with intrapulmonary metastatic lung adenocarcinoma with EGFR exon 19 deletion (Table 1). He achieved a clinically significant response for 10 months through the use of icotinib (Figure 1). After further progression, repeat biopsy showed the transformation from adenocarcinoma to combined SCLC. Immunohistochemical (IHC) results showed positive EGFR expression in the adenocarcinoma but negative in SCLC (Figure $2 A, 2 B$ ), and the positive $\mathrm{RB} 1$ expression in both adenocarcinoma and SCLC (Figure 2C,2D). In July 2016, pathological biopsy showed the pure SCLC, with EGFR exon 19 deletion, TP53 Y220H, and RB1 F755V mutations (Table 1). The IHC results showed the decreased EGFR expression and negative RB1 expression in SCLC (Figure 2E,2F). From July to December 2016, the patient received combined radioactive particle implantation and 6 cycles of chemotherapy of irinotecan plus cisplatin, and achieved complete response for 8 months. Until March 2017, computed tomography (CT) showed increasing metastatic lung lesions, but biopsy did not detect tumor cells. In July 2017, the patient exhibited enlarged intrapulmonary metastatic lesions and 2 cycles of paclitaxel plus cisplatin were administered. In October 2017, the disease had rapidly progressed. The patient stopped taking icotinib and underwent 4 cycles of paclitaxel plus cisplatin. After 3 months, CT revealed progression of the lung lesions and new hepatic metastases. The pathological biopsy showed SCLC with EGFR, TP53 Y220H, and RB1 F755V mutations (Table 1). The patient received apatinib treatment, but stopped after 10 days owing to hemoptysis. 


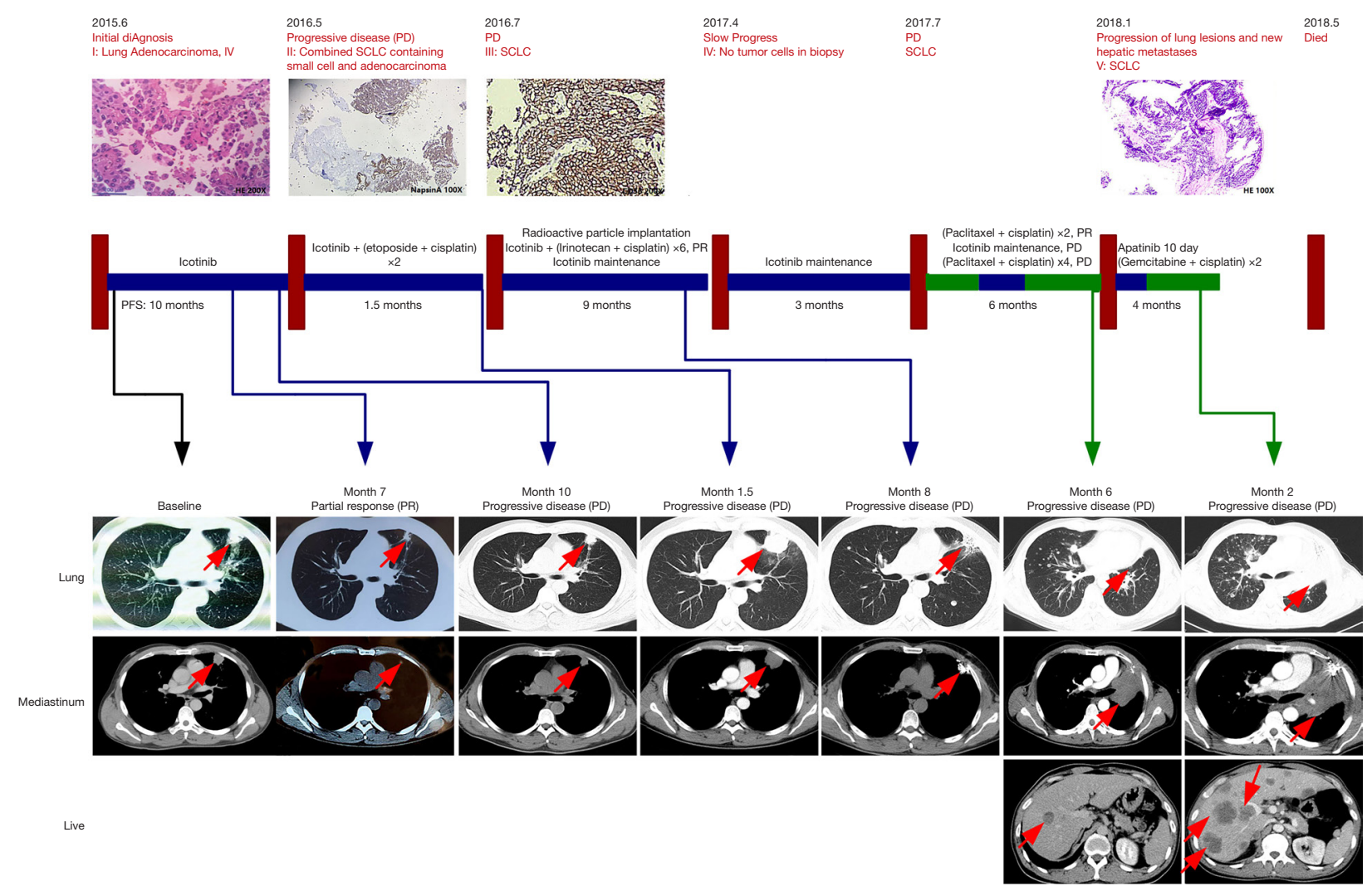

Figure 1 A summary of patient's treatment history and clinical response to the treatment in each clinical stages. The red arrow indicates the location of the tumor. Magnification: 200x in 2015.6 HE stain and 2016.7 CD56 IHC, 100× in 2016.5 Napsin A IHC and 2018.1 HE stain. HE, hematoxylin and eosin; Napsin A, napsin A aspartic peptidase; CD56, recombinant cluster of differentiation 56.

After 2 cycles of gemcitabine plus cisplatin, the patient developed multiple intracranial metastases. Unfortunately, the patient died of aggressive cancer progression on 10 May 2018. During treatment, the serum tumor levels of neuronspecific enolase (NSE) and carcinoembryonic antigen (CEA) were elevated (Figure 3).

All procedures performed in this study were in accordance with the ethical standards of the institutional and/or national research committee(s) and with the Helsinki Declaration (as revised in 2013). Written informed consent was obtained from the patient for publication of this case report and accompanying images. A copy of the written consent is available for review by the editorial office of this journal.

\section{Discussion}

Transformation from NSCLC to SCLC is a potential mechanism of acquired EGFR-TKI resistance. There are several hypotheses about the mechanism of transformation from adenocarcinoma to SCLC. First, both adenocarcinoma and SCLC were coexist in tumor samples, while the adenocarcinoma component was controlled by EGFR-TKI and the SCLC component assumed dominance (7). Second, cancer stem cells that had originally differentiated into adenocarcinoma differentiated into SCLC after external pressure such as EGFR-TKI (8). It has been reported that transformation from NSCLC to SCLC rarely occurs in non-EGFR-mutant NSCLC treated by chemotherapy (9). These studies implied that the transformation of NSCLC to SCLC may associated with the external pressure of different treatments. Third, the conditional knockout of TP53 and RB1 resulted in the development of SCLC (10). $\mathrm{RB} 1$ and $\mathrm{p} 53$ were inactivated in the both NSCLC and the transformed SCLC, but active in SCLC. It is speculated that SCLC transformation may be a clonal evolutionary event of NSCLC cells, which depends on the activation state of $\mathrm{RB} 1$ and $\mathrm{p} 53$. In this case, the pathological type showed 

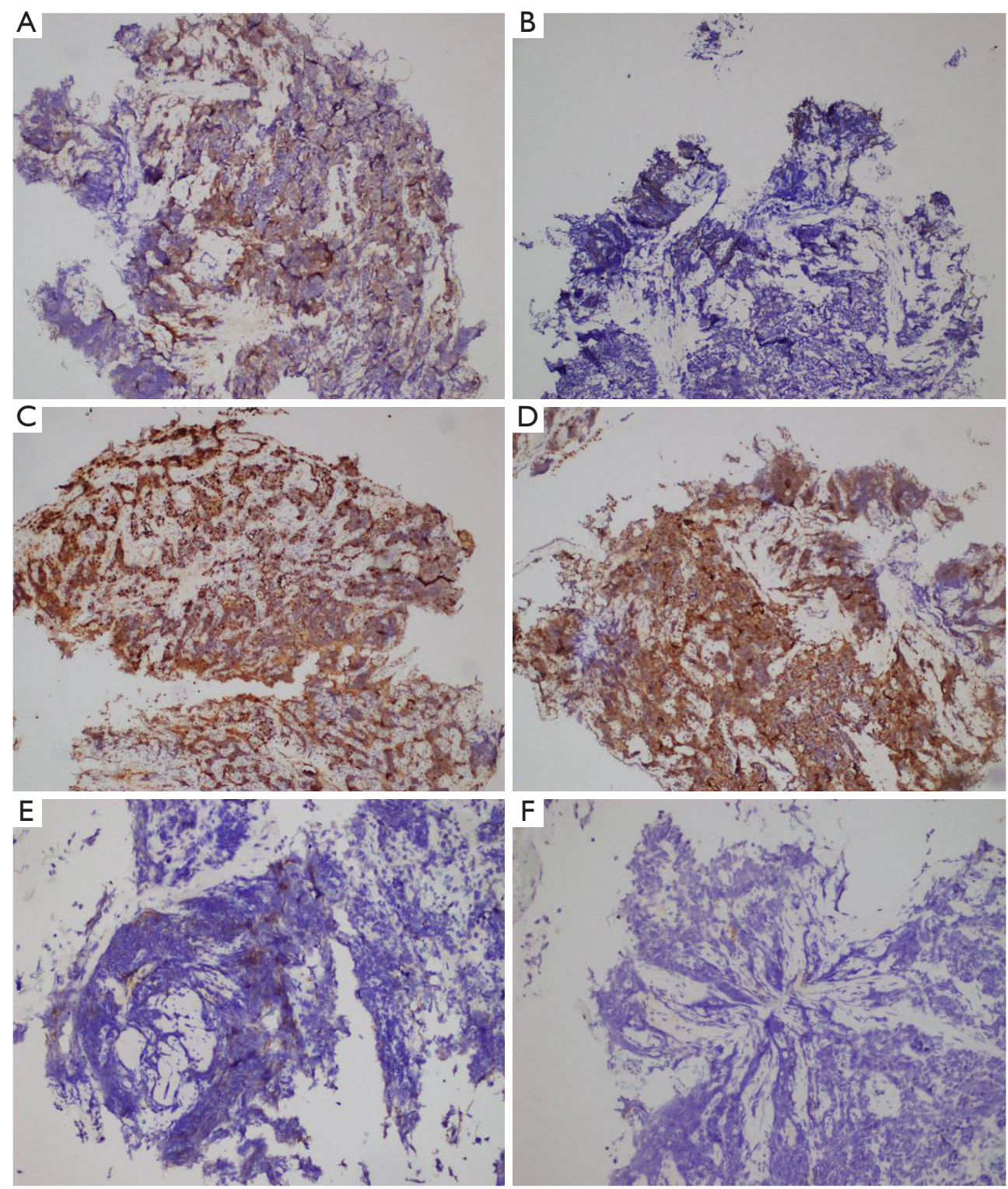

Figure 2 IHC detection of RB1 and EGFR. (A) EGFR was positive in adenocarcinoma from the second biopsy samples. (B) EGFR was negative in SCLC from the second biopsy samples. (C) RB1 was positive in adenocarcinoma from the second biopsy samples. (D) RB1 was positive in SCLC from the second biopsy samples. (E) Radioactive particle implantation of EGFR in SCLC from the third biopsy samples. (F) RB1 was negative in SCLC from the third biopsy samples. Magnification: $100 \times$ in (A), (B), (C), and (D), 200× in (E) and (F). IHC, immunohistochemical; RB1, RB transcriptional corepressor 1; EGFR, epidermal growth factor receptor; SCLC, small cell lung cancer.

a transformation from NSCLC to SCLC after EGFRTKI treatment, accompanied by EGFR, TP $53 \mathrm{Y} 220 \mathrm{H}$, and $R B 1 \mathrm{~F} 755 \mathrm{~V}$ mutations. Confirmed by IHC, the RB1 F755V mutation may result in RB1 loss in SCLC, which may contribute to SCLC transformation. Furthermore, the detection of ETV6-NTRK3 fusion, EGFR amplification, and CCNE1 amplification implied the possible mechanism of

\section{EGFR-TKI resistance.}

Transformed SCLC is similar to classic SCLC considering the genomic and morphological findings, clinical symptoms, and drug sensitivity (11). Most patients with SCLC show a clinically significant response to the treatment of etoposide plus cisplatin (12). Considering EGFR sensitive mutations, EGFR-TKI is supposed 


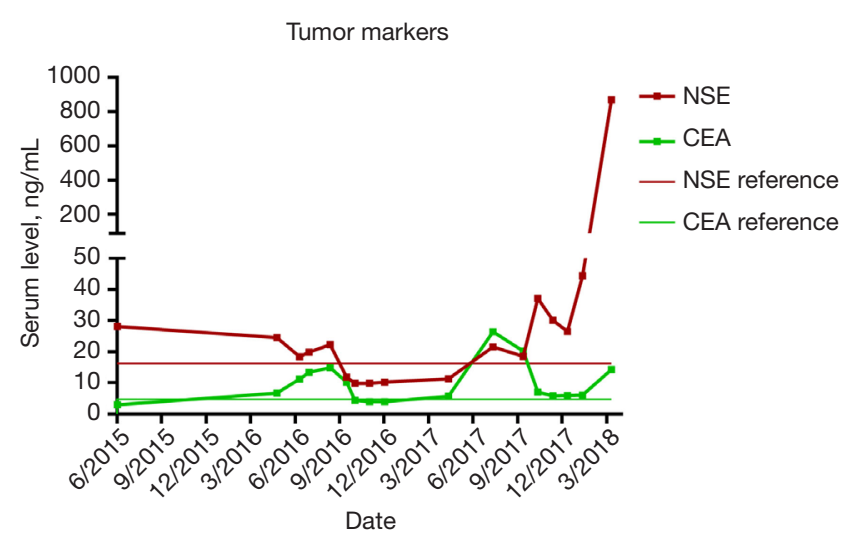

Figure 3 The change of NSE and CEA level during the treatment. NSE, neuron-specific enolase; CEA, carcinoembryonic antigen.

to be effective for NSCLC-transformed SCLC (13). Studies have shown different effects of EGFR-TKIs on transformed SCLC. One patient received treatment with gefitinib plus etoposide, and achieved a progression-free survival for 6 months (14), while another patient received afatinib treatment but showed disease progression after 2 months (15). Although the sensitive mutations of EGFR persisted, the decreased expression of EGFR may lead to the EGFR-TKI resistance in transformed SCLC (16). In this case, the EGFR expression was positive in adenocarcinoma component but negative in SCLC component. For EGFR sensitive patients failed with previous EGFR-TKI treatment, clinical trial showed that the combination strategy of carboplatin paclitaxel combined with atezolizumab and bevacizumab has well effect (17). In addition, erlotinib combined with cisplatin treatment also stabilized the condition of patients with transformed SCLC (18). However, whether to continue to EGFRTKI treatment depends on the specific clinical efficacy. We suggest that it is necessary to re-biopsy the progressed or recurrent tumor to determine the characteristics of transformed SCLC, so as to provide basis for further treatment.

Neuron-specific enolase (NSE) and carcinoembryonic antigen (CEA) are 2 major serum tumor markers of lung cancer. The increased serum NSE level is associated with SCLC. In this case, the NSE level was $28.19 \mathrm{ng} / \mathrm{mL}$ at the first visit, which was higher than the normal level. Although no SCLC component was detected on pathological biopsy, the existence of SCLC at initial diagnosis could not be excluded. During treatment, the serum NSE and CEA levels were consistent with the treatment effects. These results suggest that the elevated NSE level may indicate a high tendency of transformation to SCLC. Nevertheless, additional studies are required to confirm the usefulness of these tumor markers.

In conclusion, the possibility of SCLC transformation should be considered when patients develop acquired resistance to EGFR-TKIs. Repeated biopsy, next-generation sequencing (NGS), and IHC are necessary to identify the changes in the histological type, genetic evolution, and protein expression, after which the appropriate treatment should be determined.

\section{Acknowledgments}

Funding: This project was supported by a grant from the 2017 Hebei Province Program for Clinical Medical Talent.

\section{Footnote}

Reporting Checklist: The authors have completed the CARE reporting checklist. Available at https://atm.amegroups. com/article/view/10.21037/atm-21-6991/rc

Conflicts of Interest: All authors have completed the ICMJE uniform disclosure form (available at https://atm. amegroups.com/article/view/10.21037/atm-21-6991/coif). XS reports that he is an employee of OrigiMed Co. Ltd, Shanghai, China. The other authors have no conflicts of interest to declare.

Ethical Statement: The authors are accountable for all aspects of the work in ensuring that questions related to the accuracy or integrity of any part of the work are appropriately investigated and resolved. All procedures performed in this study were in accordance with the ethical standards of the institutional and/or national research committee(s) and with the Helsinki Declaration (as revised in 2013). Written informed consent was obtained from the patient for publication of this case report and accompanying images. A copy of the written consent is available for review by the editorial office of this journal.

Open Access Statement: This is an Open Access article distributed in accordance with the Creative Commons Attribution-NonCommercial-NoDerivs 4.0 International License (CC BY-NC-ND 4.0), which permits the noncommercial replication and distribution of the article with the strict proviso that no changes or edits are made and the 
original work is properly cited (including links to both the formal publication through the relevant DOI and the license). See: https://creativecommons.org/licenses/by-nc-nd/4.0/.

\section{References}

1. Wang M, Chen M, Shi Y, et al. P2. 02-018 Genomic Profiling of Driver Gene Mutations in 498 Chinese NSCLC Patients. J Thorac Oncol 2017;12:S2105.

2. Gao G, Ren S, Li A, et al. Epidermal growth factor receptor-tyrosine kinase inhibitor therapy is effective as first-line treatment of advanced non-small-cell lung cancer with mutated EGFR: A meta-analysis from six phase III randomized controlled trials. Int J Cancer 2012;131:E822-9.

3. Tan CS, Gilligan D, Pacey S. Treatment approaches for EGFR-inhibitor-resistant patients with non-small-cell lung cancer. Lancet Oncol 2015;16:e447-59.

4. Shinohara S, Ichiki Y, Fukuichi Y, et al. Squamous cell carcinoma transformation from adenocarcinoma as an acquired resistance after the EGFR TKI therapy in (EGFR-mutated) non-small cell lung cancer. J Thorac Dis 2018;10:E526-31.

5. Roca E, Gurizzan C, Amoroso V, et al. Outcome of patients with lung adenocarcinoma with transformation to small-cell lung cancer following tyrosine kinase inhibitors treatment: A systematic review and pooled analysis. Cancer Treat Rev 2017;59:117-22.

6. Lee JK, Lee J, Kim S, et al. Clonal History and Genetic Predictors of Transformation Into Small-Cell Carcinomas From Lung Adenocarcinomas. J Clin Oncol 2017;35:3065-74.

7. Yano S, Wang W, Li Q, et al. Hepatocyte growth factor induces gefitinib resistance of lung adenocarcinoma with epidermal growth factor receptor-activating mutations. Cancer Res 2008;68:9479-87.

8. Zhang W, Li Y, Wu D. Advances in the Transformation to Small Cell Lung Cancer from Non-small Cell Lung Cancer Following Acquired Drug-resistance to EGFR Tyrosine Kinase Inhibitors. Zhongguo Fei Ai Za Zhi

Cite this article as: Fang G, Liu W, Shang Y, Huo R, Shi X, Wang Y, Li J. Characterization of non-small cell lung cancer transforming to small cell lung cancer and its response to EGFR-TKI: a case report. Ann Transl Med 2022;10(2):115. doi: 10.21037/atm-21-6991
2017;20:720-6.

9. Norkowski E, Ghigna MR, Lacroix L, et al. Small-cell carcinoma in the setting of pulmonary adenocarcinoma: new insights in the era of molecular pathology. J Thorac Oncol 2013;8:1265-71.

10. Sutherland KD, Proost N, Brouns I, et al. Cell of origin of small cell lung cancer: inactivation of $\operatorname{Trp} 53$ and $\mathrm{Rb} 1$ in distinct cell types of adult mouse lung. Cancer Cell 2011;19:754-64.

11. Niederst MJ, Sequist LV, Poirier JT, et al. RB loss in resistant EGFR mutant lung adenocarcinomas that transform to small-cell lung cancer. Nat Commun 2015;6:6377.

12. Jiang SY, Zhao J, Wang MZ, et al. Small-Cell Lung Cancer Transformation in Patients With Pulmonary Adenocarcinoma: A Case Report and Review of Literature. Medicine (Baltimore) 2016;95:e2752.

13. Oser MG, Niederst MJ, Sequist LV, et al. Transformation from non-small-cell lung cancer to small-cell lung cancer: molecular drivers and cells of origin. Lancet Oncol 2015;16:e165-72.

14. Zakowski MF, Ladanyi M, Kris MG, et al. EGFR mutations in small-cell lung cancers in patients who have never smoked. N Engl J Med 2006;355:213-5.

15. Popat S, Wotherspoon A, Nutting CM, et al. Transformation to "high grade" neuroendocrine carcinoma as an acquired drug resistance mechanism in EGFRmutant lung adenocarcinoma. Lung Cancer 2013;80:1-4.

16. Sequist LV, Waltman BA, Dias-Santagata D, et al. Genotypic and histological evolution of lung cancers acquiring resistance to EGFR inhibitors. Sci Transl Med 2011;3:75ra26.

17. Horn L, Mansfield AS, Szczęsna A, et al. First-Line Atezolizumab plus Chemotherapy in Extensive-Stage Small-Cell Lung Cancer. N Engl J Med 2018;379:2220-9.

18. Lai L, Meng W, Wei J, et al. Transformation of NSCLC to SCLC after 1st- and 3rd-generation EGFR-TKI resistance and response to EP regimen and erlotinib: 2 CARE-compliant case reports. Medicine (Baltimore) 2021;100:e25046. 